\title{
Individual differences in the vibrotactile perception of a "simple" pattern set
}

\author{
ROGER W. CHOLEWIAK and AMY A. COLLINS \\ Princeton University, Princeton, New Jersey
}

\begin{abstract}
Discriminative capacities for vibrotactile spatiotemporal patterns were examined in 62 college students with three tasks: identification, masking, and discrimination of the letters " $X$ " and "O" presented tactually on the Optacon, a reading machine for blind persons. Individual differences in performance and interrelations among scores within and across paradigms were explored. In identification, most persons quickly achieved consistently better than $90 \%$ performance, but others failed to identify the patterns above $80 \%$, even after prolonged training. The same performance variance was found when the task was repeated by $23 \mathrm{Naval}$ student pilots. Masking and discrimination measured susceptibility to interference when patterns followed one another closely in time. The resulting functions were typical, with poorest performance at short stimulus onset asynchronies. Again, a wide range of performance was seen. Individual performance, however, appeared to be consistent across tasks, suggesting that abilities in a variety of pattern-perception tasks might be predictable.
\end{abstract}

It is generally acknowledged that individual differences exist in the ability to perform tactile pattern-perception tasks. Despite the importance of this issue in both basic and applied realms, it has not been fully explored quantitatively. The few studies in this area have generally had an applied emphasis, primarily because tactile pattern perception can be used in communication aids. The largest population of persons employing some form of tactile communication systems are blind persons. Bliss (1978), for example, studied tactile pattern processing in a large group of blind users of the Optacon, a reading machine that provides vibrotactile spatiotemporal patterns of alphabet letter shapes. He reported a wide range in ability, as measured through self-reported reading rates after varied periods of natural experience with the device. In another study of reading rates on the Optacon, Goldish and Taylor (1974) reported data from a large group (107 users), but these rates also were established only by self-report. Controlled studies, in which pattern acquisition as a function of experience is measured, are rare in the literature and typically involve only a few subjects (e.g., Linvill \& Bliss, 1966, who describe learning curves from only 3 subjects, as does Craig, 1978b). After Craig (1977) described individual data from 2 observers whose pattern perception appeared to be extraordinary, he surveyed a large number

This study and preparation of this manuscript was supported by NIH Grant DC 00076 and ONR Grant N00014-95-1-0387 to Princeton University. The authors would like to express their appreciation to Christine D. Hakami and Shannon K. Merrill for their assistance in collecting data in these experiments, and to Carl E. Sherrick for his constructive comments on the manuscript. The authors also wish to thank the editor and three anonymous reviewers for their helpful criticism of an earlier version of the paper. Requests for reprints may be sent to the authors at the Department of Psychology, Green Hall, Princeton University, Princeton, NJ 08544-1010 (e-mail: rcholewi@phoenix. princeton.edu). of persons for similar exceptional perceptual abilities (Craig, 1978a, 1983, 1988). In these studies, he did not characterize different individual abilities other than to describe them as exceptional or normal, reporting neither the ranges nor the characteristics of the tactile processing of these persons. Similarly, in the course of examining perceptual learning in a group of individuals using tactile patterns on the Optacon, Epstein, Hughes, Schneider, and Bach-y-Rita (1989) found two distinct patterns of results indicating that some naive subjects were "learners" and some were "nonlearners" (their descriptors). The $30 \%-40 \%$ of the group that were nonlearners were distinguished by the fact that they rarely exceeded chance performance on tasks in which they were to learn to identify each of eight abstract shapes moving across the finger in different directions at different rates. Interestingly, the errors made by the two groups were surprisingly similar, suggesting similarities in perceptual processing between groups as well as a common influence of shape and structure in determining a pattern's confusability.

The results of these studies argue for a more specific study of tactile pattern acquisition in a relatively large group of individuals, in which performance is recorded over the whole period of training. The intention in the present study was to provide such data, with a somewhat simple pattern set. Participants would serve in identification tasks in which only two tactile patterns, presented individually, would have to be learned. It was anticipated that considerable differences in performance might be found in even such a "simple" task over an unselected population. However, tactile experiences typically involve having to rapidly and accurately process strings of such patterns, presented in close temporal proximity to one another. Consequently, additional testing would involve discrimination and masking paradigms with pairs of these patterns, with the intention of examining the influence of temporal fac- 
tors on performance. Studies have shown that even under the best of conditions, streams of information tend not to be processed quickly when presented tactually. Braille reading rates, for example, normally range up to 80 $100 \mathrm{wpm}$ (with compressed Braille) and on the Optacon are rarely higher than $40-50 \mathrm{wpm}$ even with considerable practice (Kay, 1984). These rates might be limited for a number of reasons. For example, haptic patterns interfere with one another spatially and temporally when presented in series. By this interaction, the perceived intensity of the target stimuli may be considerably reduced, the apparent location of one or the other stimulus might be perturbed, or the spatial features of patterns could be distorted (e.g., Cholewiak, 1976; Craig, 1982; Craig \& Evans, 1987; Evans, 1987; Geldard, 1982). How an individual's perceptual system handles patterns presented close together in time has been shown to be related to other tactile pattern-perception tasks. As mentioned above, Craig (1977) examined the performance of 2 observers who could read strings of vibrotactile patterns at a rate of $100 \mathrm{wpm}$ with minimal training. In his study of their extraordinary ability, Craig found that the only measure of pattern processing that seemed to discriminate these exceptionally good observers from the norm was their resistance to tactile masking. These findings suggest that the ability to process the members of a string of stimuli depends on how susceptible a person is to interference from the preceding or following ones. The present study explored how several aspects of tactile pattern processing were related to one another to disclose whether there was a consistent pattern of performance that persisted over paradigms and task demands. The consistency of the interrelations should indicate whether similar faculties underlie a number of pattern-processing abilities that could allow for prediction of performance on more complex tasks. In addition, such data would address practical issues such as the extent of training that might be required of a population of users of tactile aids that utilized such spatiotemporal patterns.

The potential population of individuals who might be candidates to use tactile communication systems is large and diverse (e.g., Geldard, 1974). There are a number of reasons why a person might have a need for such a device. For example, a sensory disability that deprives an individual of information that cannot easily be replaced by the other senses (e.g., blindness or deafness) may require that the sense of touch be recruited (Bilger, 1977; Craig, 1976; Green \& Craig, 1982; Heller \& Schiff, 1991; Kay, 1984; Sherrick, 1984; Summers, 1992). Similarly, there are a number of conditions in which the so-called major senses are overloaded or compromised. These include space flight and underwater navigation in which the individual can readily lose personal orientation because the cues typically available, either through vestibular and/ or visual modalities, may be absent or contradictory (Howard, 1986; Howard \& Templeton, 1966). Even in normal aircraft flight, certain maneuvers result in proprioceptive information that may conflict with that available visually, leading to confusion and possible inappropriate ac- tion on the part of the pilot. Tactile displays may provide the means for displaying such data in a less ambiguous fashion (e.g., Mead, Rupert, \& Jarmul, 1994; Rupert, Guedry, \& Reschke, 1993).

As varied as the reasons are for tactile display systems, they will inevitably interact with the potential user at the man-machine interface. In this interaction, individual characteristics of the user may ultimately determine the success or failure of the device. Whether the user is a deaf person augmenting the information available in lipreading with a vibrotactile aid, a blind person converting printed text with a tactile reading machine, or a pilot being presented with changing orientation or position information, a similar process for incorporating the information available in the display streams takes place. The present study should provide an indication of the individual characteristics that might predict success with such tactile aids. The need for this is clear from a survey of screening procedures used to evaluate potential users of one widely available tactile aid, the Optacon, in research and commercial settings. There is a paucity of any reliable methods or consistent data that would indicate the probability of success an individual might have in processing rapidly changing strings of such vibrotactile patterns (Hall, 1980; Hislop, Zuber, \& Trimble, 1983; Plant, 1992; Tobin \& James, 1974). Several studies have explored the variability in tactile pattern processing in specific populations (e.g., diabetics-Harley, Pichert, \& Morrison, 1985; veterans-Gadbaw, Dolan, \& De l'Aune, 1977; primary- and secondary-school students - Weisgerber, Everett, Rodabaugh, Shanner, \& Crawford, 1974), but, as mentioned above, few have examined a general population in a well-controlled way.

In this paper, we examine the processes of learning and acquisition of vibrotactile patterns, with particular focus on the performance of individual observers and the variability in their performance in an elementary set of tasks, including three paradigms: identification, masking, and discrimination. In the series of studies, we evaluate each observer's pattern-processing capability using a set of two 2-dimensional patterns presented on the Optacon array. Learning is tested with patterns presented individually and in combination at different display rates to determine the discriminative capacity of each individual as well as his/her susceptibility to the effects of masking stimuli, which appear to be related to optimal levels of tactile pattern perception (Craig, 1977).

An additional factor to be explored was prompted by the fact that two different situations usually exist in the introduction of individuals to the use of tactile aids. Either the person has some awareness of the appearance of the patterns to be presented, and thus may have preconceived notions of what they might feel like (typical of persons who become blind late in life, or in some research situations) or the person may not know what the pattern "looks" like (e.g., individuals processing tactual analogs of acoustic stimuli). In the latter case, similar to that experienced by the congenitally blind person who must learn to use a tactile aid, patterns have to be appre- 
ciated solely on the basis of information gained tactually. These two conditions, operationally implemented by either providing individuals with visual representations of the patterns to be judged tactually or not doing so were examined in this study in order to evaluate the influence of such processes as visual imagery on tactile pattern perception.

\section{METHOD}

\begin{abstract}
Apparatus
In all of the experiments to be described, the patterns were presented to the pad of the left index fingertip. Stimuli were presented to this site with the vibrotactile array from a reading machine for blind persons, the Optacon RID (Telesensory Systems, Inc., Mountain View, CA). The Optacon provides for presentation of dense tactile patterns with a 144 vibrator ( 6 column $\times 24$ row) array that measures $12.7 \times 29.2 \mathrm{~mm}$ (Cholewiak \& Collins, 1990; Craig \& Sherrick, 1982). The vibrators in the rows are centered $1.27 \mathrm{~mm}$ $(50 \mathrm{mil})$ from one another, while those in the columns are centered $2.54 \mathrm{~mm}$ ( $100 \mathrm{mil})$ from one another. However, because the array is longer tha1 the average fingerpad, the patterns were constructed so that they would use only the upper 18 rows of the 6 columns. The patterns were presented with the 230 -pps characteristic vibratory frequency of the Optacon display. A custom-designed printer-port interface allows for control of pattern generation on the Optacon with an IBM-PC/XT-compatible computer. In an extension of the identification experiment, a portable testing system employing a laptop computer, with the same interface, was used.
\end{abstract}

\section{Subjects}

The subjects in this study were 29 male and 33 female Princeton University students. They ranged in age from 18 to 33 years $(M=$ $22, S E=.40$ ), were recruited by poster or word-of-mouth in the Princeton Department of Psychology, were paid for their participation, and they had no previous experience with vibrotactile pattern perception tasks. Forty-two of these subjects also participated in an experiment on vibrotactile threshold at the index fingertip, reported by Goble, Collins, and Cholewiak (1996), either before or immediately after being tested in the present study.

In an extension of the identification experiment, an additional 23 subjects were tested at the Naval Air Station in Pensacola, Florida. These individuals were Navy or Marine student pilots ( 21 males, 2 females), 22-25 years old ( $M=23.26, S E=0.21$ ), recruited through the Naval Aerospace Medical Research Laboratories (NAMRL).

\section{General Procedures}

The series of tests with vibrotactile patterns included discrimination, identification, and masking of a set of two patterns, the letters " $X$ " and " $O$," presented on the vibrotactile array of the Optacon. In one condition, the subjects were not told the identity or shown the visual shapes of the patterns until the end of the experimental series. It was assumed that this condition would be similar to that encoun- tered by blind individuals attempting to learn to identify pictorial vibrotactile patterns. In the other condition, pictorial representations were viewed prior to the first experiment, a procedure that is typical of the majority of published experimental studies. In addition, the order of the three tasks within the series varied for these two conditions for reasons of testing convenience. The discrimination task was either first or third in the order of testing. Since it has been shown that discrimination does not appear to depend on the ability to identify the patterns (Cholewiak \& Craig, 1984; Horner \& Craig, 1989 ) and yet reveals temporal processing capacity, this variation in procedure was not expected to affect the resulting data. In this task, the subject is required to say only whether the members of a pair of stimuli are the same or different, regardless of their identity. This method has been used previously to examine processing capabilities within and across body sites (e.g., Cholewiak \& Craig, 1984; Homer \& Craig, 1989). In those studies, it was shown that it was possible for observers to perform well without necessarily being able to identify the individual patterns. One explanation for this is that subjects might be sensitive to a change in the stimulus within the pattern epoch without regard to the cause of that change (Cholewiak \& Craig, 1984). Thus, two different patterns presented in succession might produce perceived movement, or a shift in perceived intensity, or even an apparent change in size, whereas if they were identical, it would be less likely that such changes would occur. The other two paradigms, identification and masking, must be done in sequence, because subjects must be trained to identify the patterns prior to judging them in the presence of a masker. When pictorial representations of the patterns were withheld from subjects, the discrimination task was done as the first task in the series. Of the 62 observers in the study, 27 were not told the shape of the patterns during testing; the remaining 35 were shown a drawing of the patterns to be presented on the vibrotactile array prior to the first block of trials (see Table 1).

Patterns were presented in the "static" mode, in which all of the pins that made up the two-dimensional display were turned on briefly for the entire pattern duration (in the manner of a tachistoscope), rather than in a spatiotemporal sequence. One or two patterns, depending on the paradigm, were presented for $26 \mathrm{msec}$ each ( 6 cycles of the Optacon's 230-pps waveform) within each trial. The two patterns that were used in all of the experiments were shaped like the sans serif letters " $X$ " and "O." To increase the likelihood that they would have the same apparent intensity (Cholewiak, 1979), the number of vibrators in the two patterns were identical ( 36 elements). The two patterns shared only 4 elements in common on the array. In other words, the pattern communality or percent overlap was $11 \%$ (Cholewiak \& Collins, 1995). In each paradigm, a block included a randomly ordered series of 100 trials. In the paradigms in which two patterns were presented within a single trial, stimuli were separated from one another by intervals ranging from 0 to $478 \mathrm{msec}$, resulting in stimulus onset asynchronies (SOAs) of 26 to $504 \mathrm{msec}$. In such cases, trials were blocked within the session by SOA. Craig (1983; Cholewiak \& Craig, 1984) has found SOA to be the best measure of the interaction between sequentially presented patterns. The subjects were instructed to respond on a two-button

Table 1

Testing Procedures

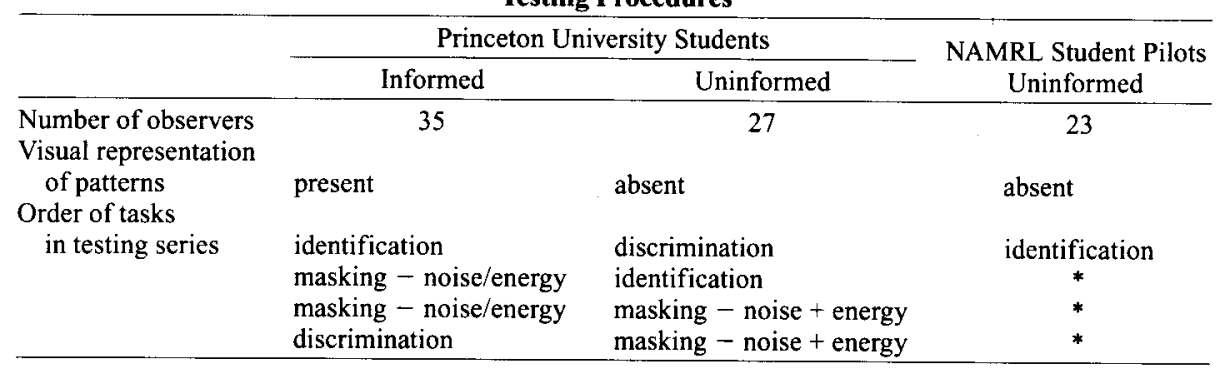


keypad with the right hand. When the paradigms were identification or masking, these keystrokes indicated the identity of the target patterns. If the subject was being tested in the condition in which no visual representation of the pattern set was given, each pattern was referred to only by the numbers " 1 " or " 2 " (encoding the letters " $X$ " and "O," respectively), and the keypad was so labeled. In the other condition, in which participants were told the shape of the patterns, the keypad was labeled with the letters " $X$ " and "O." When the discrimination paradigm was used, however, the keys encoded whether the patterns were felt to be "one and the same" (S) or "two different patterns" (D). Prior to each experiment, care was taken to ensure that the representation of the keys was clear to the subject.

The subject sat in a comfortable chair with the left arm resting on a countertop and the index finger on the vibrotactile array of the Optacon. The subjects wore headphones through which white noise was presented to minimize cues that might be available from the sound of the stimuli presented on the array and to mask any other distracting ambient noises. The array itself was covered by a 0.5 -mil plastic film to protect it from environmental contaminants. The presence of this film has been shown not to affect tactile pattern perception of the type measured in these studies (Cholewiak \& Collins, 1990). A 750-g sandbag was placed over the hand and wrist to help the observer to maintain a stable position. At the beginning of each session the experimenter read a standard set of instructions to the subject, although clarification and questions were encouraged. Prior to each block of trials, a special keystroke presented an alignment pattern (a $\Pi$ shape) to the finger to help the subject correctly position his/her fingertip on the array. During the trial series, each response initiated presentation of the next stimulus after $1 \mathrm{sec}$ of feedback and a $1-\mathrm{sec}$ ready period. Both visual and auditory feedback were provided to the subject after each trial. Specifically, high- or low-pitched tone pips in the headphones signaled correct or incorrect responses, respectively, while the correct letter or number response appeared on a visual display. An opportunity for a short rest period was provided between each block of trials within a session. At the end of each session, the visual display printed the percent correct for the series.

\section{Paradigms}

Identification. In the identification task, the patterns to be learned were presented one at a time in 100-trial blocks of 50 presentations of " $X$ " and 50 of "O." The random ordering was constrained only insofar as to ensure that the first and second halves of a block contained equal numbers of each pattern. In this paradigm, it was necessary for the subject to be able to correctly identify each pattern. A typical session involved 300-400 trials (depending on performance level) and took less than $30 \mathrm{~min}$. The criterion for terminating the training series required $85 \%$ performance in two successive 100 -trial blocks. As described above, identification was tested in two different positions within the orders of paradigms: either first in the series of three or second after discrimination. Furthermore, one group was restricted in their knowledge of the patterns to what was felt by the finger (until the end of the experimental series), while a second group was shown illustrations representing the active contactors in the two patterns.

In an extension of the identification experiment, a group of student pilots completed a single session of 300 trials (three blocks) of the identification task. They were tested in Pensacola, Florida, but the same procedures as described above were followed in this replication. All participants in this group were uninformed, and so were restricted in their knowledge of the pattern set to the information that they could gain through their tactual experience, as was true for one of the Princeton University subject groups.

Masking. Masking was always tested immediately after subjects had mastered the pattern-identification task. This paradigm involved trials in which the identity of a pattern (either " $X$ " or " $O$ ") was judged when paired with a masking stimulus. The subjects were told which one of the patterns in the pair, distinguished by its position in the time series, was to be identified (the target) while the other pattern (the masker) was to be ignored. In this masking series, the target was always the first pattern in a pair. This design is known as backward masking, which typically produces a larger effect than forward masking (Cholewiak \& Collins, 1988; Craig, 1982; Evans, 1987).

There were two types of nontarget patterns, one made up of randomly selected subsets of pins on the array, varying throughout the pattern epoch (dynamic tactile noise masker; see, e.g., MacKay, 1965 ; Uttal, 1969), and the other made up of every pin on the array turned on during the masker's 26-msec duration (energy masker). These two types of masker were chosen because they had been shown to produce two different patterns of results that were presumed to reveal separate underlying processes (e.g., Craig, 1982). Evans and Craig (1986) have shown that energy maskers tend to interact at short SOAs with target patterns so as to produce integration responses that involve components of both target and masker stimuli, while data from this laboratory (Cholewiak \& Collins, 1988) indicate that these processes are absent when dynamic noise maskers are used. Second, because of the difference in the number of active pins ( $30 \mathrm{vs} .108$ ), the dynamic noise stimulus is perceived to be somewhat less intense than the energy mask (Cholewiak, 1979; Cholewiak \& Collins, 1990), and masking has been shown to be a function of masker intensity. The differences in the maskers would allow for an examination of these two types of influence on perception of patterns which might vary in their effect for different individuals. For example, it is possible that integration might extend over longer SOAs for individuals who are less skilled at pattern identification, resulting in greater susceptibility to the disruptive influence of the energy mask.

Subjects served in two sessions. Each consisted of five blocks of 100 masking trials, distinguished by SOA, and one block of 100 trials in which no masker was presented (an identification series, to monitor the baseline level of performance). Each session contained only one masker type for the informed group, while both masker types were randomly mixed throughout sessions for the uninformed group. This procedural difference was a matter of testing convenience and was expected to have no effect on the data. In each trial, subjects were presented with one of the two target stimuli, separated from the $26-\mathrm{msec}$ masker by an interstimulus interval that resulted in SOAs of 26, 52, 104, 208, or $504 \mathrm{msec}$. The subject was reminded of the direction of masking on the visual display at the beginning of each session (e.g., TARGET FIRST), and was informed about how quickly one pattern would follow the other (SOA) prior to each block, using descriptive adjectives, such as FAST or SLOW (or IDENTIFICATION if only the target was to be presented). The subjects were unaware of which masker type was present on any trial. Furthermore, the character of the masking stimuli was described in only the broadest terms (e.g., referred to as "blobs"). Subjects who did not learn the individual patterns to criterion in the identification series were not tested here. In addition, for scheduling reasons, some subjects were not tested with the energy masker. In total, there were 50 subjects in the energy-masking condition and 58 in the noisemasking condition.

Discrimination. In the discrimination task, pairs of patterns were presented with SOAs of 26,52,104, 208, and $504 \mathrm{msec}$, randomly ordered over blocks. All combinations of the " $X$ " and " $O$ " patterns were presented as target stimuli within a trial. In half of the trials, both patterns were the same; in the remaining trials, they were different. Consequently, there were four possible stimulus patterns $\mathbf{X}-\mathbf{O}, \mathbf{O}-\mathbf{X}, \mathbf{X}-\mathbf{X}$, or $\mathbf{0}-\mathbf{O}$. In the informed group, the subjects were told that the patterns to be discriminated were " $X$ " and " $O$," and the resulting four combinations that would be presented were described. The uninformed group was given no information about the patterns to be presented. The subjects responded by indicating only whether the stimuli presented were both the same or of two different patterns. A discrimination session involved five blocks of 100 trials, and took less than $45 \mathrm{~min}$. At the beginning of each block, SOA was indicated on the visual display with a descriptive adjective (e.g., FASTEST). In this task, the subjects did not have to identify the indi- 
vidual patterns in order to correctly discriminate between them. Because the responses in this case were "same" or "different," all individuals were included, even those who did not learn to identify the target patterns to criterion.

\section{RESULTS AND DISCUSSION}

\section{Identification}

The data for each observer from each block of trials were collapsed into a $2 \times 2$ stimulus-response matrix across patterns. Due to the possibility of response bias, a total corrected-percent-correct, $P(C)_{\max }$, was calculated from this matrix with a computer algorithm (see Cholewiak \& Craig, 1984, for details). This statistic minimizes the effects of any such bias (McFadden, 1970). It should be noted that the differences between the values of percent correct obtained from the raw data versus $P(C)_{\max }$ were typically less than $2 \%$. As described earlier, two different procedures were employed in the identification task, one in which the subject knew the visual representation of the patterns and one in which he/she did not. Analysis of variance (ANOVA) of the performance demonstrated that there was no significant difference in the identification data between the two procedure groups $[F(1,60)=0.705, p>.05]$. The combination of the availability of a visual representation of these patterns and the position of the identification task within the testing series did not appear to affect identification performance. Accordingly, the identification data collected with both procedures were combined for the analyses to follow. These results are consistent with the findings of Terzieff, Stagg, and Ashcroft (1982), who described the acquisition of reading skills on the Optacon with different training methods, and also found little effect of procedure. In addition, it is notable that Epstein et al.'s (1989) subjects always had available visual representation of the patterns that they were judging, yet almost half of these persons were unable to learn to identify the stimuli.

Mean identification performance is shown in Figure $1 \mathrm{~A}$ in the form of a learning curve in which $P(C)_{\max }$ is plotted as a function of sets of 50 trials (dividing the actual 100-trial blocks in half in order to examine performance changes more carefully). Means in later sessions include extrapolated final levels from individuals who achieved criterion early in the series. The figure shows that the mean performance on this task (connected points) is quite good, exceeding the $85 \%$ criterion level within the first 100 trials. Although most subjects did quite well on this task, a wide variation in performance was apparent. To illustrate this, the individual performance for each observer is plotted adjacent to the means for the $1 \mathrm{st}$, 10th, and 20th blocks (the remaining points, omitted for clarity, had comparable extents). The range of performance observed in these initially naive subjects is somewhat remarkable considering the apparent simplicity of the task. One might think that the two patterns could have been identified by any number of cues, especially given the trialby-trial feedback. Yet, differences in performance among individuals were evident early within the first block of 100 trials, necessitating analysis in 50-trial sets. As the figure shows, the majority of subjects eventually learned to identify the patterns. A large number of participants ( 34 of 62) needed to serve in only the minimum number of two blocks (200 trials) before reaching criterion (85\% correct or better on two consecutive blocks of 100 trials). In fact, within the first 50 trials, some observers ( 25 of 62) had scores above $95 \%$. Several others ( 4 of 62 ), however, did not reach the performance criterion, and 2 never exceeded $80 \%$ correct even after relatively prolonged training involving 10 blocks ( 1,000 trials). (The $85 \%$ criterion level is shown on the graph by a small-dashed line, while chance performance is shown by a larger dashed line.)

To illustrate that this performance range is not unique to this subject population, another experiment was conducted in which Navy and Marine student pilots were tested at the Naval Aerospace Medical Research Laboratories in Pensacola, Florida. Their performance over the course of three blocks (six 50-trial increments) is shown in Figure 1B, along with the Princeton student data from Figure 1A, replotted for comparison. Aside from the fact that every observer in this smaller group exceeded $85 \%$ correct by the third and final block, the means for the two populations are virtually identical: ANOVA shows that there are no significant differences between the groups $[F(1,83)=0.328, p>.05]$. Furthermore, the performance ranges of the two groups in the first and following sessions were comparable (e.g., ranges in the first 50-trial block were: Princeton students, 47\%-100\%; NAMRL student pilots, $48 \%-100 \%$ ). These data provide for another important comparison. Recall that there was a difference in experience between the Princeton informed and uninformed groups (see Table 1), so our conclusions regarding the influence of presence of a visual representation had to be qualified owing to its covariation with experience. The NAMRL pilot group, however, like the Princeton informed group, were tested on pattern identification without any previous experience of feeling spatiotemporal stimuli on the Optacon. Because the NAMRL group was uninformed, they provide a more valid comparison of the effect of visual representation on pattern identification without the confound of experience. ANOVA shows that there are no significant differences between the Princeton University informed and the NAMRL uninformed groups $[F(1,56)=0.024, p>.05]$. If the comparison is made between the NAMRL uninformed group and the Princeton uninformed group, allowing the effect of order with identical conditions of visual representation to be examined, no significant differences are found between the groups $[F(1,48)=1.025, p>.05]$.

When individual learning curves for this task were visually examined, it became apparent that the data were clustered into four performance groups that could be distinguished on the basis of how well and how quickly individuals learned to identify the patterns. This was true even of the subjects who met the criterion by exceeding $85 \%$ correct within the first two blocks in the experiment: Some subjects achieved performance above $95 \%$ within the first 50 trials (and were labeled as "Group 1"); others 


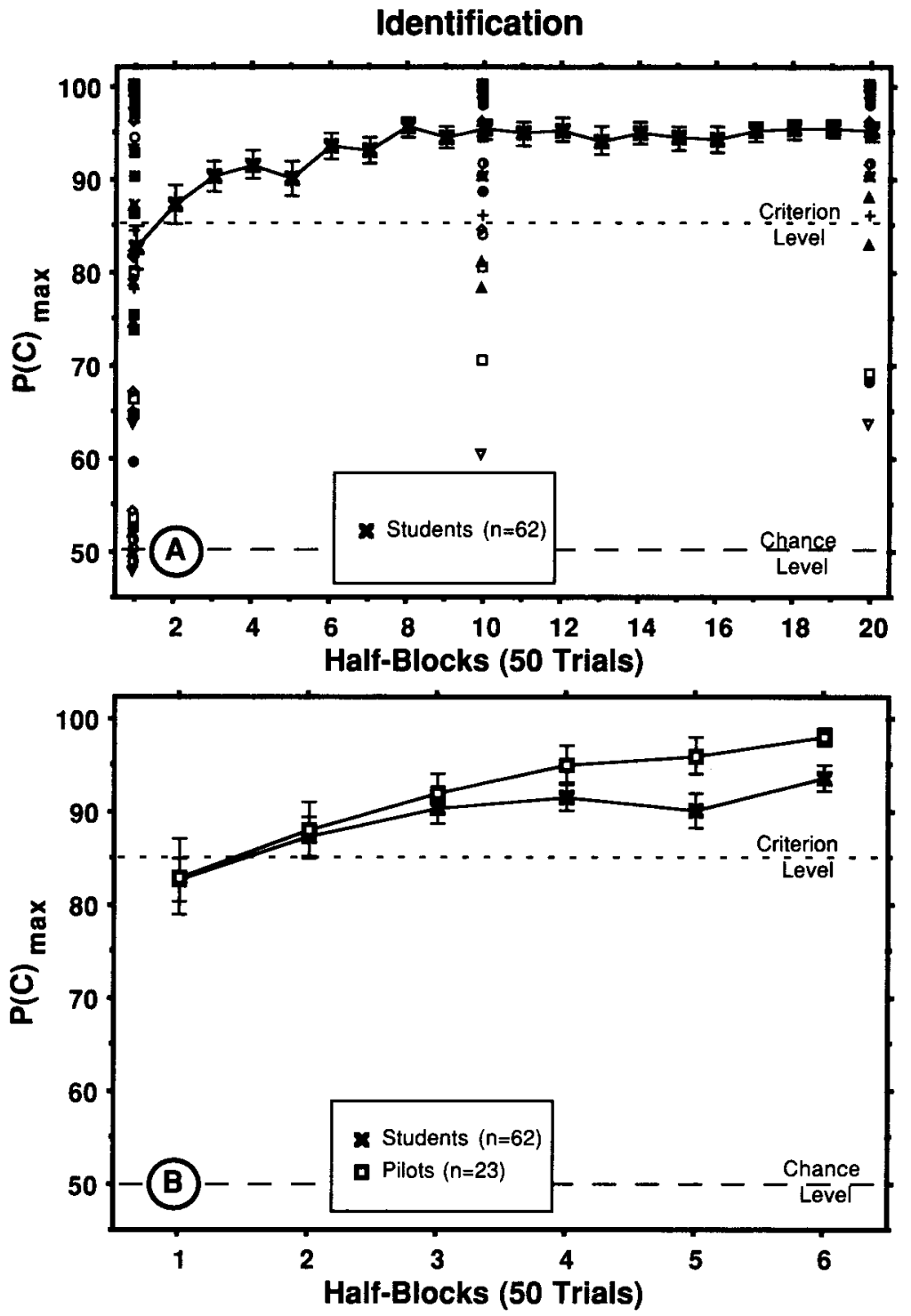

Figure 1. (A) Vibrotactile pattern-identification performance for a group of initially naive college students over the course of 1,000 trials. The range of performance is shown by the individual data points shown at three representative levels of training. Chance performance (50\%) is shown by the large-dash line in this and the following figures, while the criterion performance level $(85 \%)$ is indicated by the small-dash line in these illustrations. Means in later sessions include extrapolated final levels from those individuals who achieved criterion early. (B) Identification performance of 23 student pilots from the Naval Aerospace Medical Research Laboratories in Pensacola, Florida, over the course of 300 trials of training/testing on the "X-O" pattern set, with performance of the college students over this period replotted from (A) for comparison. Note a change in the scale of the abscissa from that in (A). Standard errors of the means are shown about the data points.

did not (labeled as "Group 2"). Still other subjects needed additional blocks of trials to meet the $85 \%$ correct criterion, yet met it within 1,000 trials (Group 3). Finally, the performance of a few persons did not meet the criterion within the 1,000-trial extent of the study (Group 4). The merit of these groupings is that they reflect a combined measure of both rate of acquisition and terminal performance level. Figure 2 shows the learning curves for each group. Because Group 1 individuals learned so quickly, comparative data exist across all four groups only for the first four sets of 50 trials (identification training was discontinued after the performance criterion was reached). A between-groups ANOVA over the first four sets of 50 trials found that the performance means of these groups were significantly different from one another $[F(3,58)=$ $61.584, p<.01]$. In addition, there is a significant inter- 
action between performance group and experience with the task (number of 50-trial sets) $[F(9,174)=3.500, p<$ $.01]$, indicating that the shape of the learning curves for the four groups indeed are different, perhaps most evident in the fact that the performance of Group 1 subjects ceases to improve after the first 50 trials (a ceiling effect because of their superior performance), while the performance of other subjects improved with additional trials.

Although there was variation in the amount of training needed, the majority of the subjects were able to learn to identify the two patterns used in the identification task: Only four participants fell into Group 4. This group is similar to Epstein et al.'s (1989) "nonlearners" who, despite extended training, never learned to recognize scanned patterns on the Optacon at rates much above chance levels. There is, however, a difference in number. Forty-two percent of their 24 participants were nonlearners, whereas only $6 \%$ failed to learn the patterns in the current study. The reason for this difference between studies may be procedural. First, no feedback was provided in Epstein et al.'s (1989) study, so only incidental learning may have occurred in this group, suggesting an inability to locate and retain in memory discriminable features in the pattern set. Second, although Epstein et al. employed only eight asymmetric shapes as stimuli, when the several velocities, directions, and display-window widths are taken into account, the total number of different spatiotemporal patterns to be identified numbered almost 200 . In the course of the experiment, these were presented only four times each. Certainly, some generalization could occur across rates and window widths, but the task is still not as simple as initially appears. Given the complexity of this task, and the results from the present study, it is possible that the "nonlearners" in Epstein et al.'s study may be individuals who, like the members of our Groups 2 and 3 shown in Figure 2, would master the set given extended training. In summary, it is likely that a task involving additional trials, trial-by-trial feedback, as well as a restricted pattern set (reducing the trial-to-trial variability in stimuli), as was used in the present study, would provide the poorer subjects with a better chance for success.

These data illustrate the diversity in performance across a relatively uniform subject population, selected only on the basis of admission to the University (or to a student pilot program). Whatever the constellation of criteria that determines adequacy for matriculation, they do not guarantee success on the apparently uncomplicated task of identifying the members of a pair of vibrotactile patterns that, in one case, encircle the fingerpad while, in the other case, fill the center of the same pad. The next experiment examined whether the differences in acquisition described above by the performance group persist into a task involving a temporal dimension, and in which it is assumed that the participants have been trained to a criterion ability to identify the two "simple" patterns.

\section{Masking}

As in the case of the identification experiment, ANOVAs found that there was no significant difference between the two different procedure groups (informed or uninformed about the pattern shapes) in the ability to identify these patterns under masking $[F(1,48)=3.752, p>.05]$. A significant interaction between procedure, SOA, and masker type did appear $[F(4,192)=4.413, p<.01]$, apparently

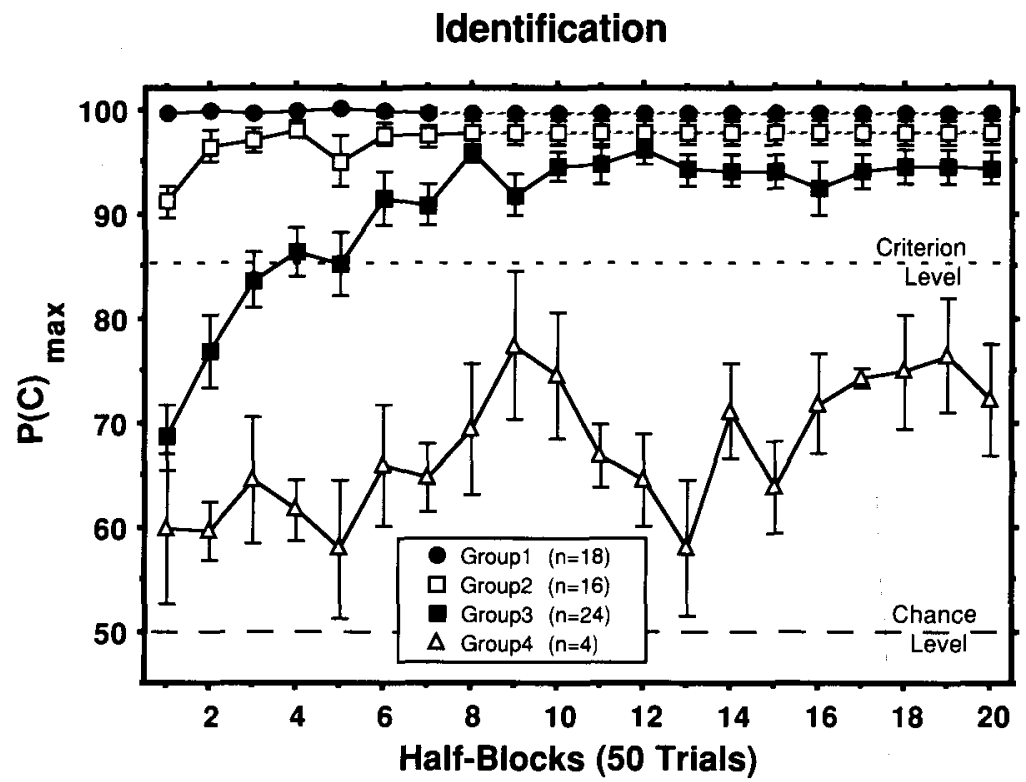

Figure 2. Vibrotactile pattern-identification performance for the group of initially naive college students over the course of 1,000 trials, plotted with performance group as the parameter. The selection procedure is described in the text. Dotted lines between points indicate extrapolations from the final block for subjects who achieved criterion early in testing. Standard errors of the means are shown about the data points. 
the result of the fact that performance levels for the informed and uninformed groups at 26-msec SOA are more similar to one another with the energy masker than with the noise masker. This result is difficult to interpret, given the lack of a main effect of procedures. Consequently, it seems that providing or withholding a visual representation of the patterns to be identified and the placement of the masking task within the testing series did not influence performance in an important way. This was not unexpected, given that subjects were required to reach criterion identification performance prior to serving in this experiment, and so variation in training methods should not affect posttrained performance. Because training procedure appears to have had little effect on masking performance, the data from the two training conditions were combined for the analyses to follow.

The data from the masking study are shown in Figure 3, with masker type as the parameter. As expected, the longer the time between masker and target, the better the targetidentification performance. The effect of SOA is significant $[F(4,192)=102.728, p<.01]$, a result typically found in tactile pattern-masking studies (Craig, 1980). From these data it also appears as though the energy mask is slightly less effective in disrupting performance than is dynamic tactile noise. The direction of the difference in masker effectiveness in itself is somewhat unusual. It was expected that the presence of the energy masker would result in significantly poorer performance than when the noise masker was used, particularly because the two masker types were not equated in perceived intensity. (In direct comparisons, the energy mask is perceived to be noticeably more intense than the noise mask; see, e.g., Cholewiak \& Collins, 1988, 1990; Craig, 1982; Craig \& Evans, 1987.) Nevertheless, although the difference between masker types is small, it is significant $[F(1,48)=11.332, p<.01]$, with dynamic noise producing more masking than the energy stimulus. This finding is also contrary to the hypothesis presented earlier that the energy mask might affect performance more because of its greater propensity to involve more complex integrative processes. These occur in experiments in which geometric forms are masked: The energy mask has been found to be more effective than noise, even when the two maskers are equated in perceived intensity (Cholewiak \& Collins, 1988). This effect appears to have been particularly influential when the predominant features in the targets occupied the edges of the display, loci optimally affected by the rectangular energy mask. A possibility in the present study is that the crucial area, allowing differentiation between the " $X$ " and " $O$ " patterns, is the central region of the display, where the dynamic noise mask might be more effective.

When subjects were grouped according to their performance on the identification task, performance in the masking paradigm followed the previously established hierarchy for both masker types, as shown in Figure 4. The best performance over SOA in the presence of either masker was from subjects in Group 1, followed by those from Groups 2 and 3 , in that order $[F(2,47)=7.933, p<$ .011 . The Group 3 subjects, who, as a group, took longer to learn the patterns initially, were more susceptible to masking: Their data show the largest difference between the masked and unmasked conditions. Careful examination of the functions in Figures $4 \mathrm{~A}$ and $4 \mathrm{~B}$ indicate that

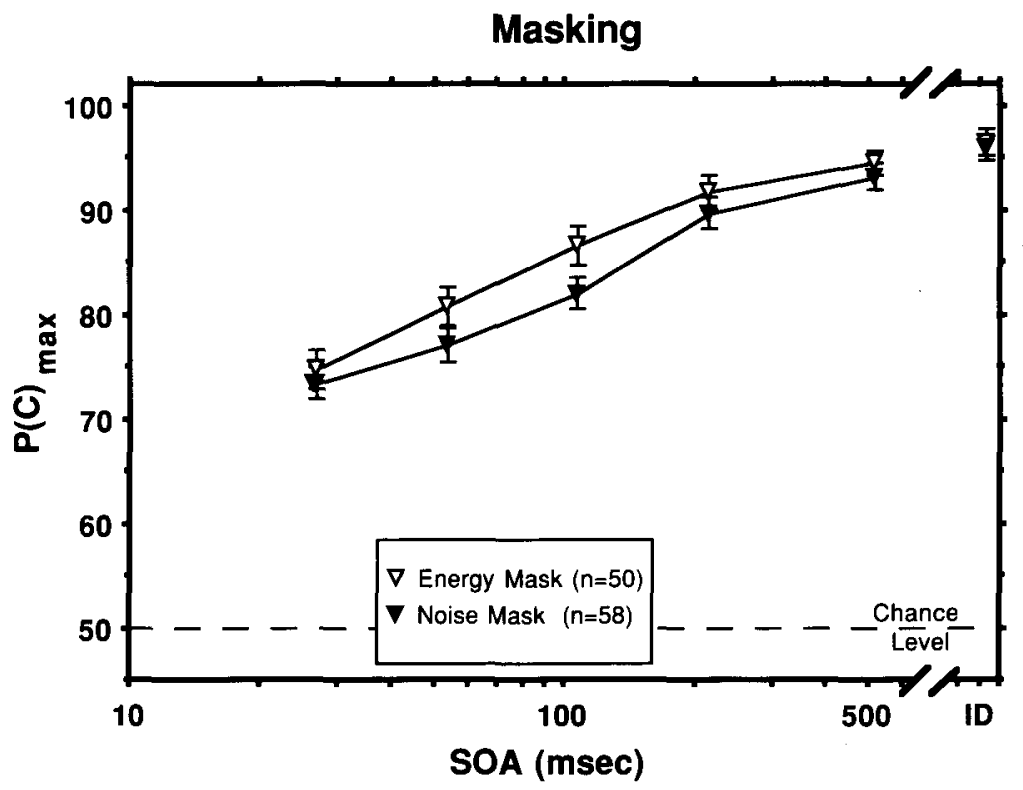

Figure 3. Masked identification of the "X-O" pattern set when the target patterns were followed by each of two masker types (shown as the parameter) with five levels of stimulus onset asynchrony (SOA). Unmasked identification performance measured within the masking series for each of the two groups is also shown. Standard errors of the means are shown about the data points. 
the overall difference in the effect of energy and noise maskers seen in Figure 3 appears primarily to be the result of the contribution of Groups 1 and 2. For these two groups, energy masking was less effective (resulting in better performance) than noise masking. This was not the case with the members of Group 3: their performance did not differ between the dynamic noise masker and the energy mask (compare the panels in Figure 4). Indeed, there is a significant interaction between masker and performance group $[F(2,47)=3.227, p<.05]$.

Recall that, overall, identification performance was poorer for Group 3 than for the other groups even after the $85 \%$ criterion was reached (Figure 2 ). It is possible that the differences in masking performance could be the result of this inequality. Consequently, the individual data were normalized to the optimal performance achieved during masking by a ratio method (i.e., performance/best performance for condition). Typically, best performance was achieved during the identification block, although some observers showed slightly higher scores during masking at an SOA of $504 \mathrm{msec}$ (most evident for Group 1 under noise masking). This could have been the result of learning or fatigue during the experimental session. In general, the pattern of results followed that of the nonnormalized data described earlier. That is, the greater effectiveness of the noise masker over the energy masker remained $[F(1,47)=12.519, p<.01]$, as did the strong effect of $\operatorname{SOA}[F(4,188)=98.184, p<.01]$, while there was still no effect of procedure on these results $[F(1,47)=$ $12.519, p<.01]$. Normalized mean data for performance groups are plotted in Figures 5A and 5B for energy and noise maskers, respectively. These figures again show that

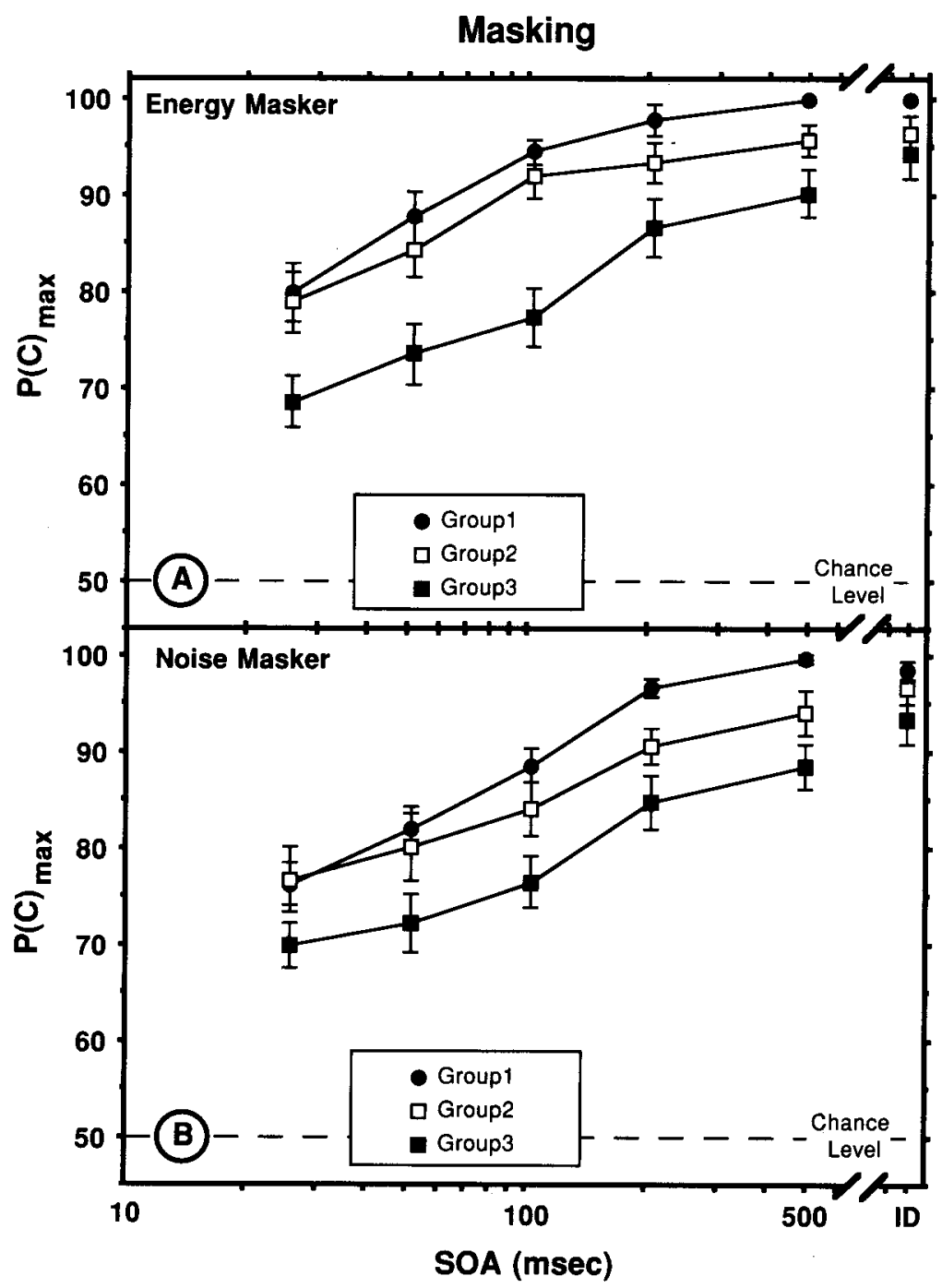

Figure 4. Masked identification of the " $X-O$ " pattern set when the target patterns were followed at five levels of stimulus onset asynchrony (SOA) by (A) energy and (B) noise maskers, plotted with performance group as the parameter. Performance in an unmasked identification block for each of the groups is also shown. Standard errors of the means are shown about the data points. 
Group 3 performance was more affected by the presence of a masking stimulus, and that the noise pattern was a more effective masker $[F(2,47)=8.132, p<.01]$, supporting the notion that susceptibility to masking is related to poor pattern-perception performance.

\section{Discrimination}

The ability to resolve vibrotactile patterns presented in close temporal proximity was also tested within a patterndiscrimination paradigm, in which subjects had to determine whether two patterns presented successively on the Optacon display were the same or different. In contrast to the other tasks, a significant difference was found between the discrimination-performance data from the informed and uninformed procedure groups. Subjects who had been presented with an illustration of the patterns to be discriminated (informed group) were significantly better able to determine whether the same or different patterns were presented than were subjects who were not shown the pictorial representations (uninformed group) $[F(1,60)=40.066, p<.01]$. Thus, withholding representations of the patterns and placing the discrimination task first in the test series resulted in a significant decrement in discrimination performance. Recall that an assumption was made that placement of a task within the testing series would have no effect on the resulting data. This assumption was based upon the findings that discrimination performance is independent of the ability to identify the patterns to be discriminated (e.g., Cholewiak \& Craig, 1984; Horner \& Craig, 1989). Thus, the significant difference between procedural groups found here is interpreted to be the result of the effects of the presence or

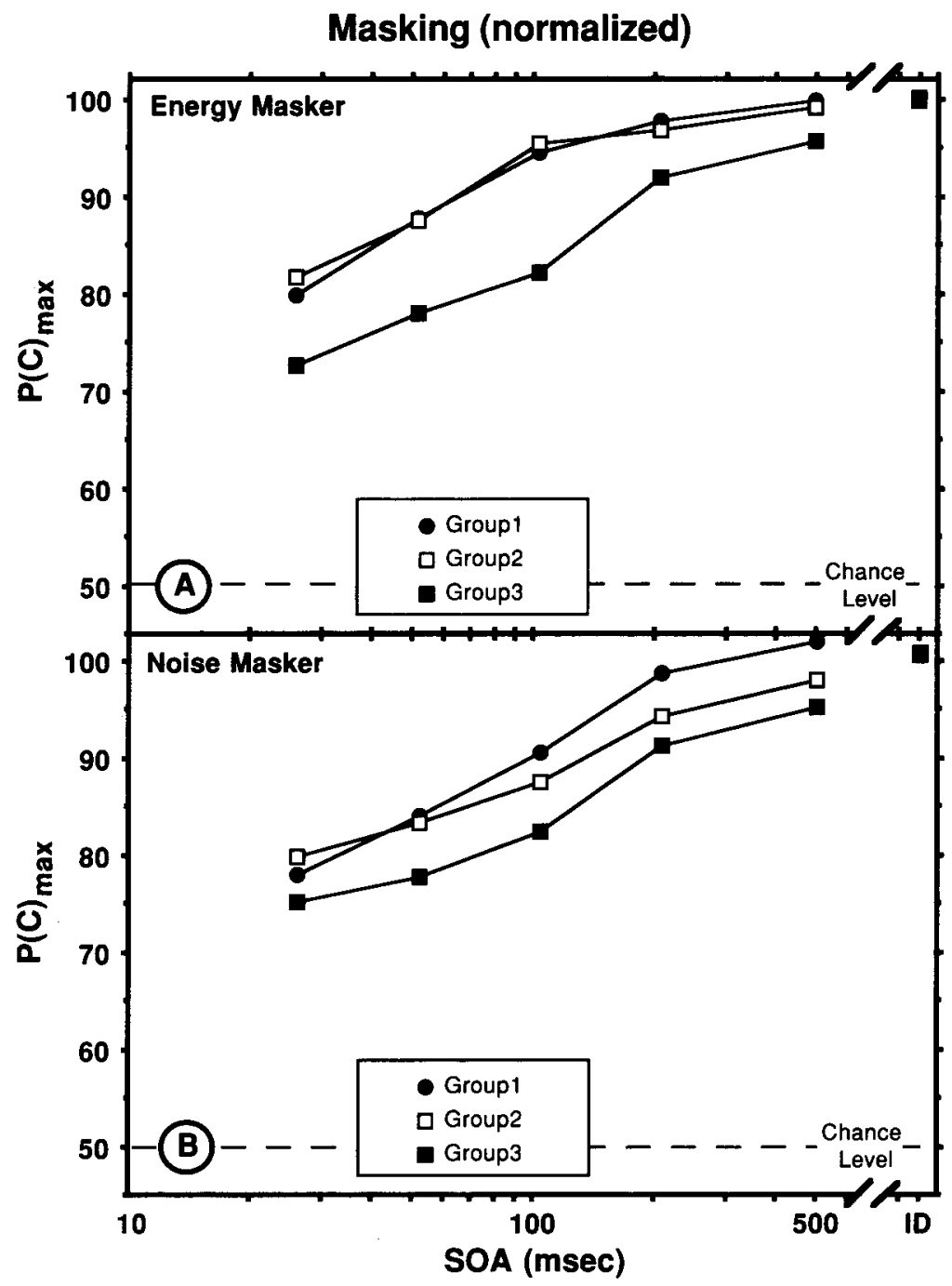

Figure 5. Masked identification of the "X-O" pattern set, shown in Figure 4, is replotted with the functions normalized to identification-block performance. Functions for (A) energy and (B) noise masking are plotted with performance group as the parameter. Normalization allows a direct comparison of the size of the masking effect for each group by eliminating the confounding influence of different initial identificationperformance levels. 
absence of the visual representation of the pattern set, despite the confound with experience, due to the fact that the uninformed group did the discrimination task first while the informed group did the task last.

In the studies from the literature described above, in which discrimination appeared to be independent of identification, subjects were shown the shapes of the members of the pattern set in use. Perhaps that information alone allowed them to direct their attention to key aspects of the stimulus presentation, resulting in better performance. It is also true that the informed group tested here knew that there were only four pattern combinations possible, whereas the uninformed group in the present study was not given this information. It is possible that subjects in the informed group performed better than those in the uninformed group, because they attempted to learn the differences among the four different trial types. However, it seems more likely that knowledge of the pattern set allowed subjects to better use emergent phenomena resulting from the rapid presentation of two stimuli, such as perceived movement, a shift in perceived intensity, or an apparent change in shape or size, that could contribute to high levels of discrimination performance in the absence of pattern identification (Cholewiak \& Craig, 1984; Mahar \& Mackenzie, 1993).

The mean discrimination-performance values for the two procedural groups are shown in Figure 6 as a function of SOA. In addition to the effect of visual representation described above, there is a significant effect of SOA $[F(4,240)=41.120, p<.01]$. Plotted for comparison in the figure are discrimination data collected by Craig and Cholewiak (1984) with a group of subjects who were both practiced and informed, under circumstances highly similar to those described for the informed condition in the present study. Their subjects achieved levels virtually identical to those of our informed group of subjects. What accounts for the poor discrimination performance of both procedure groups, particularly at brief SOAs? It is likely that masking plays a strong role in the accuracy of these judgments (see Figures 3-5; Horner, 1995; Horner \& Craig, 1989). As SOA increases, it is assumed that masking decreases and the features upon which discrimination decisions are based become more apparent so that performance improves. It is also interesting to note that even when the two patterns are separated by $504 \mathrm{msec}$, performance is still far from perfect. (Horner, 1995, describes discrimination levels continuing to be less than $100 \%$ even out to $1,000 \mathrm{msec}$ ). At these longer SOAs, this might be due to the poor nature of memory for tactile patterns, which presumably is necessary for a comparison to take place (Geldard, 1970; Millar, 1974; Murray, Ward, \& Hockley, 1975), or may be the result of some long-term effects of masking (Craig, 1982).

To examine whether individual differences are apparent in discrimination, these data were replotted in Figures $7 \mathrm{~A}$ and $7 \mathrm{~B}$, with the data divided into the earlier determined performance groups as the parameter for both informed and uninformed procedure groups. Again, despite the apparent simplicity of the task, subjects show quite different levels of performance, differentiated well by the

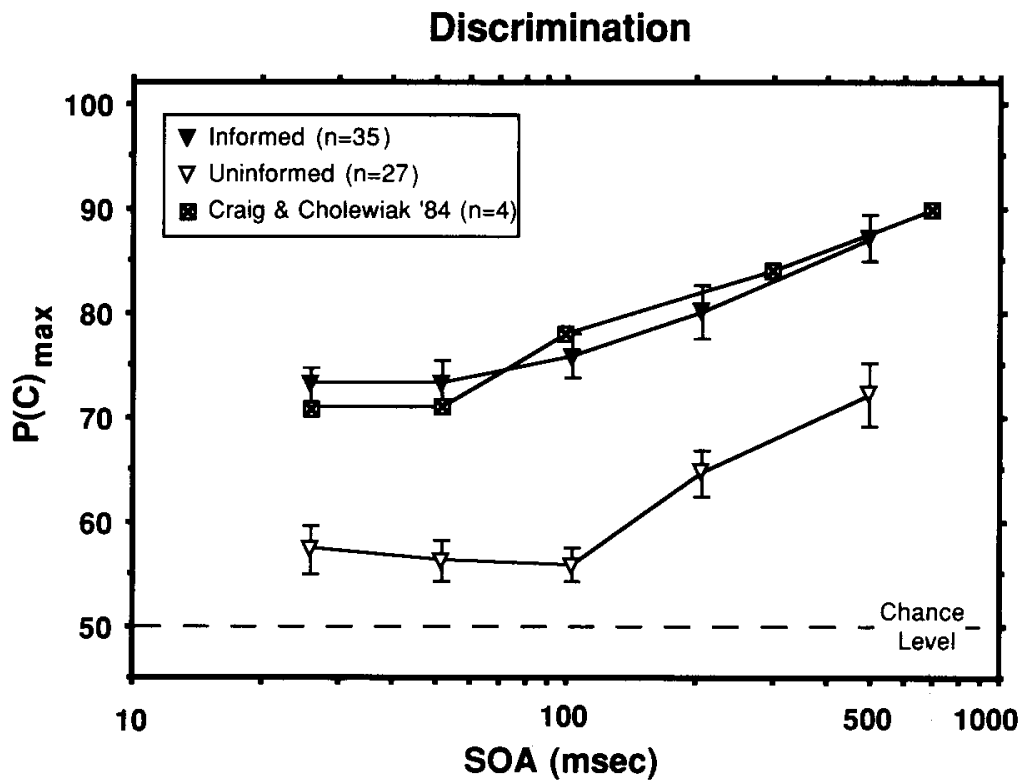

Figure 6. Discrimination data as a function of stimulus onset asynchrony (SOA) with procedure group as the parameter. Procedure groups differed in the amount of information available about the pattern set. In the informed procedure, observers saw visual representations. In the uninformed procedure, patterns were not shown. Data from Craig and Cholewiak (1984) represent the performance of subjects who were shown visual representations in a comparable task, included for comparison. Standard errors of the means are shown about the data points. 
rate and terminal levels of performance achieved in the identification task, regardless of the availability of a visual representation of the patterns to be judged. Note that, as was the case with the test of pattern identification, Group 4 performance does not go much above the level of chance even at the longest SOAs, whereas Group 1 performance in both cases is close to $90 \%$ at the longest SOA. There appears to be a clear separation between the different performance groups except at a few SOAs for Groups 1 and 2 (not unlike the similarities and differences in the masking data described earlier). The performance-level hierarchy is again consistent with the identification data showing a significant effect of group $[F(3,54)=16.684, p<.01]$.

\section{Correlational Analyses}

The pattern of results for each task, when examined according to performance group, follows that of the identification data: The relative performance levels of the groups were maintained through the series. The indication is that there is a consistency in individual performance levels in vibrotactile pattern perception that extends beyond the initial test of identification into tasks involving temporal distinctions, such as masking and discrimination. It appears as though the differences observed among participants in their initial performance levels in the relatively simple identification task pervade their performance in other similar tasks. The consistency of individual performance, within each performance group and across the series of experiments was further examined through a correlational analysis of these data. It should be noted that since the number of subjects in each task was not the same (e.g., Group 4 subjects did not complete the masking task), a series of correlational matrices were calculated, each maximizing either number of participants or number of measures compared. One such correlation matrix, typical of the others, is shown in Table 2.

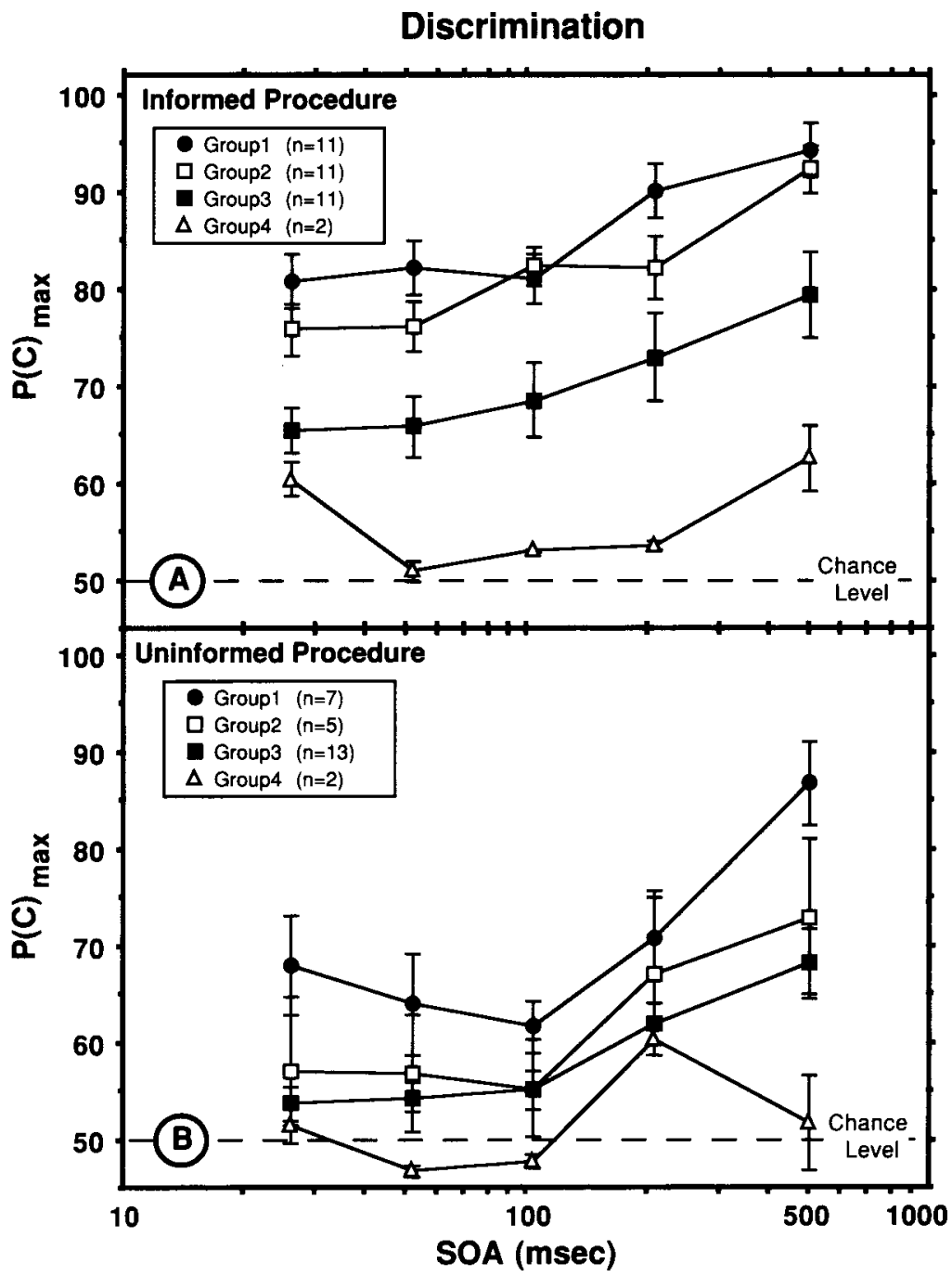

Figure 7. Discrimination of the " $X-O$ " patterns as a function of stimulus onset asynchrony (SOA) are plotted with performance group as the parameter. In the two panels are plotted the data $(A)$ for the informed procedure and $(B)$ for the uninformed procedure. Standard errors of the means are shown about the data points. 
The measures included in Table 2 were chosen on the basis of the following considerations. First, to evaluate the largest number of conditions, data from the Group 4 individuals $(n=4)$ were not included: because of their poor identification performance, they did not serve in the masking studies. In addition, another 8 persons, who did not serve in the energy-masking task because of scheduling problems, were not included in the table calculation. Furthermore, normalized masking data were used in these calculations (although examination of the results with nonnormalized data showed no important differences in the pattern of relationships). It was felt that these transformed data better represented the underlying influences of masking because they were independent of differences in absolute identification performance level. Discrimination data were not included in the table because the two procedure groups had significantly different performance levels for this task. Finally, the number of participants was limited to those whose vibrotactile thresholds had been measured within the Goble et al. (1996) study, so that the relationship of pattern-processing performance with sensory factors could be evaluated. Vibrotactile thresholds were measured at 10 frequencies over the range of 10 to $400 \mathrm{~Hz}$, but the data for only 4 representative frequencies were analyzed with the pattern-perception data. Consequently, complete data for 41 individuals were included in this table $(d f=39)$. For the one-tailed test, significance at the .05 level requires a correlation coefficient greater than .260 , while highly significant results $(p<.01)$ are indicated if the coefficient exceeds .363 .

The array shown in Table 2 reflects a finding true of all of the correlation matrices calculated: Performance among the pattern-perception tasks is well correlated. The data are the individual performance scores of each subject for each task, so that a high correlation in any cell indicates the extent to which participants who did well on one task also did well on the other task. The frequency of occurrence of statistical significance in the coefficients in the cells of the matrix indicate that individuals "remain" in their relative standings throughout the series of experiments. Many of the correlations are significant, yet none are perfect. Examination of individual data from task to task revealed that there was some movement in relative performance levels for some individuals across the series. Even so, the present findings show strong support for the notion that individual differences in performance are consistent across tasks. As was evident from the graphic examination of the performance group data, group was correlated at a highly significant level with performance in the majority of subconditions for each task. The one exception is noise masking, for which performance at the shortest SOAs was not significantly correlated with group. In fact, the noise-masking correlations show the fewest significant correlations among all of the pattern-perception measures. It is perhaps not surprising that noise masking is less related to other pattern-perception tasks than is energy masking. The suggestion that masking performance may be indicative of tactile pattern-perception ability is based on the notion that it is a measure of the amount of interference that results when similar patterns follow one another in sequence. In general, the most deleterious masking effects are produced by masking with the same pattern types. For example, letters masked by letters are more poorly identified than letters masked by other stimuli (Cholewiak \& Collins, 1988; Craig, 1982, 1983). Energy maskers, due to the apparent contrast emphasis at their edges, appear to have pattern-like qualities absent in noise maskers, so may influence letter identification through processes like integration in a manner not too unlike letters themselves (Cholewiak \& Collins, 1988; Craig \& Evans, 1987).

Given the reliability of individual differences across tasks, examination of the correlations between vibrotactile threshold measures and tactile pattern performance in Table 2 reveal that these performance differences are unrelated to sensitivity. The four frequencies of stimulation were included in the table because of their hypothesized relationships to the operation of underlying receptor populations (Bolanowski, Gescheider, Verrillo, \& Checkosky, 1988; Johnson \& Hsiao, 1992), but the remaining six frequencies were similarly unrelated to pattern processing. In fact, vibrotactile thresholds for the observers included in Table 2 ranged over as much as $30 \mathrm{~dB}$ (depending on the frequency examined; Goble et al., 1996). Because the patterned stimuli were presented at a fixed physical intensity, the consequence of this variability in threshold is that these patterns probably felt much stronger to some individuals (who had lower thresholds) than to others. Nevertheless, no obvious relationship between these differences and pattern performance existed. These results support the notion that, to a large degree, if an individual can feel a pattern, they can learn to identify it. This position is not without qualification: Craig (1980), for example, has shown that pattern duration (and thus the perceived intensity of vibrotactile patterns), as well as physical intensity, can influence levels of identification performance.

There are a number of other correlations in the table that deserve specific mention. First, within this college population, neither age nor sex were related in a meaningful fashion to performance in these tasks. Also, when the performances in the individual tasks are examined, it can be seen that the subconditions of each task are internally correlated. For example, the individual scores during the first block of 50 identification trials are correlated with those during the 6 th block of 50 trials $(r=.314$, $p<.05$ ), indicating, in this case, that initial levels are predictive of those occurring later in training. Among the subconditions for each task, significant correlations exist for masking SOAs of 52, 104, 208, and $504 \mathrm{msec}$, but fewer at $26 \mathrm{msec}$. Note that this SOA is less representative of stimuli in typical communication systems because two 26-msec patterns are not separated at all from one another in time. Only at SOAs greater than the 26-msec pattern duration is there a "quiet" period between patterns, akin to the white space between printed letters.

Although not shown, correlations were separately calculated for the two procedural groups (informed and un- 


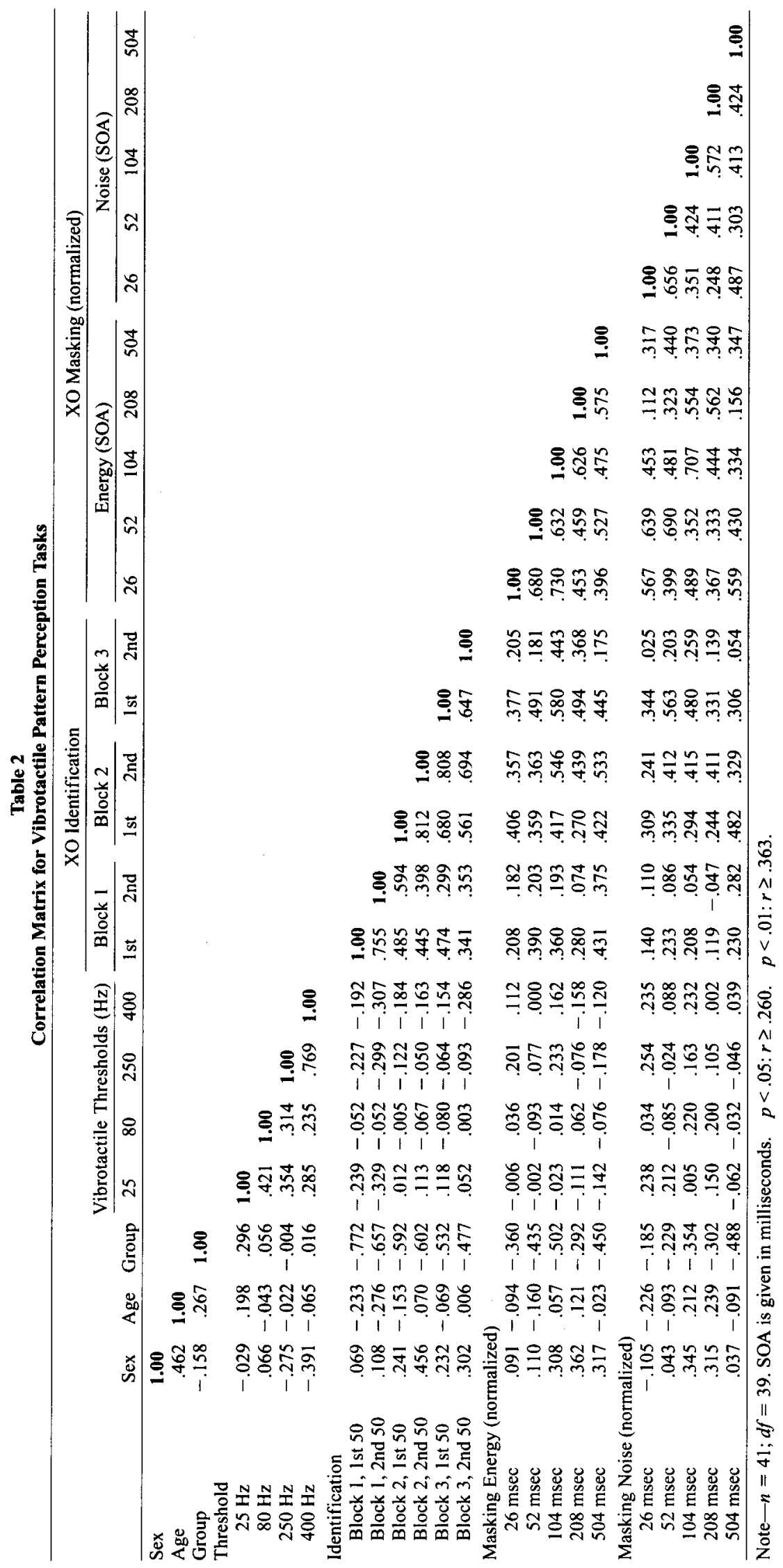


informed). These correlations incorporated all of the pattern perception data, including the results from the discrimination series. Internal consistency in individual performance over the series of tasks continued to be evident: Performance group membership and 50-trial-set identification performance were found to be highly correlated with both discrimination and masking data at most SOAs for the informed procedure group. In the case of the uninformed procedure group, however, the discrimination data showed much poorer correspondence with the other measures. These data suggest that the observer's understanding of the patterns during the discrimination task may depend on whether he/she has some appreciation of the apparent shape (as revealed through the visual representation). In addition, identification and masking data were found to be most highly correlated in the second and third blocks of identification training/testing with the uninformed group, whereas correlations between the paradigms were higher in the first session of identification training/twsting for the informed group. These results indicate that when a visual representation of the patterns is withheld, it takes somewhat more experience (50 to 100 trials more) for subjects to acquire an appreciation of the patterns that will be reflected in their ability in later pattern-perception tasks. Perhaps the additional time is needed for individuals to be able to formulate some type of internal representation of the patterns.

\section{GENERAL DISCUSSION}

These experiments have shown that individual differences in tactile pattern perception, as reflected in a number of tasks common to those required of users of tactile communication systems, appear to be large and consistent across paradigms. Some individuals (Group 1) are able to learn to identify the members of a pair of tactile patterns, sometimes within 3-5 trials. Other individuals (Group 4) may never be able to consistently recognize the patterns, even after 1,000 trials. The reliability both of individual performance and of the classification based on the first of the tests described, pattern identification, is reflected in the high correlations with other time-sensitive paradigms. These (masking and discrimination) involved patterns presented at rates equivalent to text-reading, ranging from almost $400 \mathrm{wpm}$ at $26-\mathrm{msec} \mathrm{SOA}$ to $48 \mathrm{wpm}$ at $208-\mathrm{msec}$ SOA and $20 \mathrm{wpm}$ at $504-\mathrm{msec}$ SOA, realistic levels, at least in the lower region, when one considers typical Braille-reading rates (Foulke, 1982; Nolan \& Kederis, 1969; Terzieff et al., 1982). The results demonstrate the utility of these paradigms and this pattern set in categorizing an individual's potential performance on several complex tactile pattern-processing tasks within a brief test time. This series of tasks could be useful as a screening task predictive of the processing of more complex presentations of tactile patterns. Adding to its utility, the series is quickly and easily administered: The total duration of a session of some 300 identification trials (or six 50-trial blocks) is only $20-30 \mathrm{~min}$, including orientation and instruction.
But what might be the bases of the pervasive differences found among individuals? Other sensory capabilities demonstrate considerable ranges of performance over comparable populations. For example, there are wide individual differences in situational awareness in populations of student pilots (Endsley \& Bolstad, 1994). This is the ability to retain correct appreciation of one's location in space (primarily using only vestibular, not visual, information) despite being moved passively. Such situations occur commonly in aircraft or under water. Similarly, Watson (1987, p. 272) describes a wide range in auditory discriminative capacities in a large population of normal-hearing individuals. Certainly, biological factors might account for some of this variability: One factor that might account for some of the differences in tactile pattern perception could be variations in vibrotactile sensitivity, particularly in those receptor populations that might be responsible for the encoding of these types of information (Johnson \& Hsiao, 1992). Vibrotactile sensitivity, however, does not appear to play a role, given the lack of a relationship between threshold levels and tactile pattern performance shown in the correlational analysis (Table 2). It appears that as long as patterns can be felt, they can be perceived. This is not a situation unique to tactile pattern perception: certain categories of complex visual and auditory pattern perception (such as speech sounds) are more dependent on more central cognitive mechanisms, as long as the stimuli are "within the range of peripherally-determined sensitivity and resolving power" (Watson, 1987, p. 267). Other possible physical factors, such as skin compliance and receptor density, although implicated in some types of tactile pattern perception, typically do not vary sufficiently within an age group as narrow as that explored here to explain the observed differences in pattern perception (see, e.g., Stevens \& Patterson, 1995; Woodward, 1993). Some of the performance differences among these observers may be related to the factors that appeared to contribute to the difficulty experienced by Epstein et al.'s (1989) "nonlearners" in attempting to learn to recognize an apparently simple pattern set. Epstein et al. argued, as did Loomis $(1981,1990)$, that the spatiotemporal capabilities of the learners might have matched the parameters of the test patterns better than did those of the nonlearners, so the patterns were more readily learned by these individuals.

Several findings from the present series of studies resonate with the importance of individual differences in spatiotemporal capacities, particularly those involving temporal resolution. The first indicator of the significance of temporal resolution in tactile pattern performance found in these data was the relationship, described earlier, between performance group membership and masking and discrimination performance. Pattern identification on the skin typically requires the ability to rapidly appreciate a spatial layout over a somewhat coarse sensory sheet. Unlike vision, the opportunity to scan the surface is usually not possible given the rapidity with which such patterns are presented in strings in communication systems. The observer must apprehend the whole pattern before it 
changes (in these cases, within $1 / 40 \mathrm{sec}$ ), encode or image it, put it away in some short-term store, and compare it against an internal representation in order to give it a label. Not only is this a difficult task in and of itself, but the appearance of another pattern following the first can disrupt the encoding process. The masking data suggest that individuals who were better able to identify patterns when followed by distracting stimuli also excelled in their ability to identify those patterns when presented in isolation. These results do not speak directly to the involvement of spatial factors (except by the possible involvement of distracting features from energy maskers integrating with the target stimuli; Evans, 1987), but it is similarly likely that spatial acuity plays as significant a role in individual differences as does temporal acuity (Cholewiak, 1994; Cholewiak \& Collins, 1993). One way in which this may be reflected is through the influence of visual representation on pattern perception. Knowledge of the spatial layout of the patterns did affect performance in the discrimination task.

The present study is a first step toward determining the factors that contribute to good vibrotactile patternprocessing ability in the general population. Further work in this area should concentrate on teasing out the influences of spatial and temporal acuity in the processing of pattern strings such as those employed by vibrotactile communication systems.

\section{REFERENCES}

BILGER, R. C. (1977). Evaluation of subjects presently fitted with implanted auditory prostheses. Annals of Otology, Rhinology, \& Laryngology, 86(Suppl. 38).

BLIss, J. C. (1978). Reading machines for the blind. In G. Gordon (Ed.), Active touch (pp. 243-248). New York: Pergamon.

Bolanowski, S. J., Gescheider, G. A., Verrillo, R. T., \& CheckOSKY, C. M. (1988). Four channels mediate the mechanical aspects of touch. Journal of the Acoustical Society of America, 84, 1680-1694.

Cholewiak, R. W. (1976). Satiation in cutaneous saltation. Sensory Processes, 1, 163-175.

CHOLEWIAK, R. W. (1979). Spatial factors in the perceived intensity of vibrotactile patterns. Sensory Processes, 3, 141-156.

CHOLEWIAK, R. W. (1994, March). A comparison of vibrotactile pattern perception by young and old persons. Paper presented at the workshop on Currency Features for Visually Impaired People, Washington, DC (National Research Council, National Materials Advisory Board report to the Bureau of Engraving and Printing, Department of Treasury).

Cholewiak, R. W., \& Collins, A. A. (1988). Vibrotactile pattern masking with static and dynamic tactile noise. Bulletin of the Psychonomic Society, 26, 489.

Cholewiak, R. W., \& Collins, A. A. (1990). The effects of a plasticfilm covering on vibrotactile pattern perception with the Optacon. Behavior Research Methods, Instrumentation, \& Computers, 22, 21-26.

Cholewiak, R. W., \& Collins, A. A. (1993). A comparison of complex vibrotactile pattern perception on the OPTACON by young and old observers. Journal of the Acoustical Society of America, 93, 2361.

Cholewiak, R. W., \& Collins, A. A. (1995). Vibrotactile pattern discrimination and communality at several body sites. Perception \& Psychophysics, 57, 724-737.

Cholewiak, R. W., \& Craig, J. C. (1984). Vibrotactile pattern recognition and discrimination at several body sites. Perception \& Psychophysics, 35, 503-514.

Craig, J. C. (1976). Vibrotactile letter recognition: The effects of a masking stimulus. Perception \& Psychophysics, 20, 317-326.
CRAIG, J. C. (1977). Vibrotactile pattern perception: Extraordinary observers. Science, 196, 450-452.

CraIg, J. C. (1978a). Individual differences in vibrotactile pattern recognition. Sensory World, 31, 15-17.

CraIG, J. C. (1978b). Vibrotactile pattern recognition and masking. In G. Gordon (Ed.), Active touch (pp. 229-242). New York: Pergamon.

CraIG, J. C. (1980). Modes of vibrotactile pattern generation. Journal of Experimental Psychology: Human Perception \& Performance, 6 , 151-166.

CraIG, J. C. (1982). Vibrotactile masking: A comparison of energy and pattern maskers. Perception \& Psychophysics, 31, 523-529.

CRAIG, J. C. (1983). Some factors affecting tactile pattern recognition. International Journal of Neuroscience, 19, 47-57.

CRAIG, J.C. (1988). The role of experience in tactual pattern perception: A preliminary report. International Journal of Rehabilitation Research, 11, 167-171.

Craig, J. C., \& ChOlewiak, R. W. (1984). The discrimination of vibrotactile patterns. Unpublished manuscript, Indiana University and Princeton University.

Craig, J. C., \& Evans, P. M. (1987). Vibrotactile masking and the persistence of tactual features. Perception \& Psychophysics, 42, 309317.

Craig, J. C., \& Sherrick, C. E. (1982). Dynamic tactile displays. In W. Schiff \& E. Foulke (Eds.), Tactual perception: A sourcebook (pp. 209-233). Cambridge: Cambridge University Press.

ENDSLEY, M. R., \& BoLSTAD, C. A. (1994). Individual differences in pilot situational awareness. International Journal of Aviation Psychology, 4, 241-264.

EPstein, W., Hughes, B., SChNeider, S. L., \& BaCh-Y-Rita, P. (1989). Perceptual learning of spatiotemporal events: Evidence from an unfamiliar modality. Journal of Experimental Psychology: Human Perception \& Performance, 15, 28-44.

Evans, P. M. (1987). Vibrotactile masking: Temporal integration, persistence, and strengths of representations. Perception \& Psychophysics, 42, 515-525.

Evans, P. M., \& Craig, J. C. (1986). Temporal integration and vibrotactile backward masking. Journal of Experimental Psychology: Human Perception \& Performance, 12, 160-168.

FoulKe, E. (1982). Reading Braille. In W. Schiff \& E. Foulke (Eds.), Tactual perception: A sourcebook (pp. 168-208). Cambridge: Cambridge University Press.

Gadbaw, P. D., Dolan, M. T., \& DE L'Aune, W. R. (1977). Optacon skill acquisition by blinded veterans. Journal of Visual Impairment $\&$ Blindness, 71, 23-28.

GelDARD, F. A. (1970). Vision, audition, and beyond. In W. D. Neff (Ed.), Contributions to sensory physiology (Vol. 4, pp. 1-17). New York: Academic Press.

GELDARD, F. A. (ED.) (1974). Cutaneous communication systems and devices. Austin, TX: Psychonomic Society.

GELDARD, F. A. (1982). Saltation in somethesis. Psychological Bulletin, 92, 136-175.

Goble, A. K., Collins, A. A., \& Cholewiak, R. W. (1996). Vibrotactile thresholds in young and old observers: The effect of spatial summation and the presence of a rigid surround. Journal of the Acoustical Society of America, 99, 2256-2269.

Goldish, L. H., \& TAYLOR, H. E. (1974). The Optacon: A valuable device for blind persons. New Outlook for the Blind, 68, 49-56.

GreEN, B. G., \& CraIG, J. C. (1982). Vibrotactile perception of speechderived stimuli. International Journal of Rehabilitation Research, $\mathbf{5}$, 63-66.

HALL, A. (1980). Recommendations for evaluating innovative products for the visually handicapped. Journal of Visual Impairment $\&$ Blind ness, 74, 89-92.

Harley, R. K., Pichert, J. W., \& Morrison, M. (1985). Braille instruction for blind diabetic adults with decreased tactile sensitivity. Journal of Visual Impairment \& Blindness, 79, 12-17.

Heller, M. A., \& SchifF, W. (EDs.) (1991). The psychology of touch Hillsdale, NJ: Erlbaum.

Hislop, D. W., Zuber, B. L., \& Trimble, J. L. (1983). Characteristics of reading rate and manual scanning patterns of blind optacon readers. Human Factors, 25, 379-389. 
HoRNER, D. T. (1995). A comparison of discrimination and identification of vibrotactile patterns. Perception \& Psychophysics, 57, 463-474.

HoRNER, D. T., \& CRAIG, J. C. (1989). A comparison of discrimination and identification of vibrotactile patterns. Perception \& Psychophysics, 45, 21-30.

HowARD, I. P. (1986). The vestibular system. In K. R. Boff, L. Kaufman, \& J. P. Thomas (Eds.), Handbook of perception and human performance (Vol. 2, pp. 18/1-18/62). New York: Wiley.

HoWARD, I. P., \& TEMPLETON, W. B. (1966). Human spatial orientation. New York: Wiley.

Johnson, K. O., \& HsiaO, S. S. (1992). Neural mechanisms of tactual form and texture perception. Annual Review of Neuroscience, 15 , 227-250.

KAY, L. (1984). Electronic-aids for blind persons-An interdisciplinary subject. IEE Proceedings-A, 131, 559-576.

Linvill, J. G., \& BLISS, J. C. (1966). A direct translation reading aid for the blind. Proceedings of the IEEE, 54, 40-51.

Loomis, J. M. (1981). On the tangibility of letters and Braille. Perception \& Psychophysics, 29, 37-46.

Loomis, J. M. (1990). A model of character recognition and legibility. Journal of Experimental Psychology: Human Perception \& Performance, 16, 106-120.

MACKAY, D. M. (1965). Visual noise as a tool of research. Journal of General Psychology, 72, 181-197.

MAHAR, D. P., \& MACKENZIE, B. D. (1993). Masking, information integration, and tactile pattern perception: A comparison of the isolation and integration hypothesis. Perception, 22, 483-496.

MCFADDEN, D. (1970). Three computational versions of proportion correct for use in forced-choice experiments. Perception \& Psychophysics, 8, 336-342.

Mead, A. M., Rupert, A. H., \& Jarmul, E. S. (1994). A tactile interface for virtual and dynamic environments. Proceedings of the 1994 Image VII Conference, 7, 331-334.

MilLAR, S. (1974). Tactile short-term memory by blind and sighted children. British Journal of Psychology, 65, 253-263.

MurRay, D. J., WARD, R., \& HoCKLEY, W. E. (1975). Tactile short-term memory in relation to the two-point threshold. Quarterly Journal of Experimental Psychology, 27, 303-312.

Nolan, C. Y., \& Kederis, C. J. (1969). Perceptual factors in Braille word recognition. New York: American Foundation for the Blind.
Plant, G. (1992). The selection and training of tactile aid users. In I. R. Summers (Ed.), Tactile aids for the hearing impaired (pp. 57-82). London: Whurr.

RuperT, A. H., Guedry, F. E., \& ReschKe, M. F. (1993, October). The use of a tactile interface to convey position and motion perceptions. Paper presented in the Aerospace Medical Panel Symposium at the meetings of the NATO Advisory Group for Aerospace Research and Development (AGARD), Lisbon, Portugal.

SHERRICK, C. E. (1984). Basic and applied research on tactile aids for deaf people: Progress and prospects. Journal of the Acoustical Society of America, 75, 1325-1342.

Stevens, J. C., \& PATterson, M. Q. (1995). Dimensions of spatial acuity over the life span. Somatosensory \& Motor Research, 12, $29-47$.

SUMMERS, I. R. (1992). Tactile aids for the hearing impaired. London: Whurr.

TerziefF, I., StaGG, V., \& Ashcroft, S. C. (1982). Increasing reading rates with the Optacon: A pilot study. Visual Impairment \& Blindness, 76, 17-22.

ToBIN, M. J., \& JAMES, W. R. (1974). Evaluating the Optacon: General reflections on reading machines for the blind. American Foundation for the Blind Research Bulletin, 28, 145-157.

UTTAL, W. R. (1969). Masking of alphabetic character recognition by dynamic visual noise (DVN). Perception \& Psychophysics, 6, 121-128.

WATSON, C. S. (1987). Uncertainty, informational masking, and the capacity of immediate auditory memory. In W. A. Yost \& C. S. Watson (Eds.), Auditory processing of complex sounds (pp. 267-276). Hillsdale, NJ: Erlbaum.

Weisgerber, R. A., Everett, B. E., Rodabaugh, B. J., Shanner, W. M., \& CRAWFORD, J. J. (1974). Educational evaluation of the $O p$ tacon (Optical-to-Tactile Converter) as a reading aid to blind elementary and secondary students [Final report]. Palo Alto, CA: American Institutes for Research.

WOODWARD, K. L. (1993). The relationship between skin compliance, age, gender, and tactile discriminative thresholds in humans. Somatosensory \& Motor Research, 10, 63-67.

(Manuscript received January 22, 1996; revision accepted for publication July $29,1996$. 